\title{
Review Article \\ Correlations between the Visual Apparatus and Dental Occlusion: A Literature Review
}

\author{
Alberto Baldini $\mathbb{D}^{1},{ }^{1}$ Alessandro Nota $\mathbb{D},,^{2,3}$ Silvia Caruso $\mathbb{D},{ }^{3}$ and Simona Tecco $\mathbb{D}^{2}$ \\ ${ }^{1}$ University of Rome Tor Vergata, Italy \\ ${ }^{2}$ Dental School, Vita-Salute San Raffaele University, Milan, Italy \\ ${ }^{3} \mathrm{MeSVA}$ Department, University of L'Aquila, Italy \\ Correspondence should be addressed to Alessandro Nota; dr.alessandro.nota@gmail.com
}

Received 11 December 2017; Revised 18 June 2018; Accepted 24 June 2018; Published 9 July 2018

Academic Editor: Toshiyuki Sawaguchi

Copyright (C) 2018 Alberto Baldini et al. This is an open access article distributed under the Creative Commons Attribution License, which permits unrestricted use, distribution, and reproduction in any medium, provided the original work is properly cited.

Background. The development of visual functions takes place in the first months of postnatal life and is completed around the one year of age. In this period, the maturation of the retina and the visual pathways occur, and binocular bonds are established at the level of the visual cortex. During this phase and then for a few years, a certain plasticity of the visual functions remains, which seem therefore susceptible to change both in a pejorative sense (by pathogens) and in an improving sense (for example, by therapeutic measures). This plasticity involves also the oculomotor system. Due to this plasticity, many researchers believe that there are some functional correlations between the visual and the stomatognathic apparatus. But the scientific evidence of this statement has not been clarified yet. Aim. The purpose of this review is therefore to analyze the clinical data in this field and finally to establish their level of evidence. Studies have been collected from the main databases, based on keywords. Results. The results showed a middle level of evidence since most of the data derive from case-control studies and cross-sectional studies. Conclusions. The level of evidence allows establishing that there is a correlation between ocular disorders (myopia, hyperopia, astigmatism, exophoria, and an unphysiological gait due to ocular convergence defects) and dental occlusion, but it is not possible to establish the cause-effect relationship. Future studies should be aimed at establishing higher levels of evidence (prospective, controlled, and randomized studies).

\section{Introduction}

The visual apparatus consists of equal and symmetrical organs located, for the great part in the anterior region of the head, below the forehead, and at the sides of the nose root [1].

Among these organs, the main one is the eye; the others, called accessory organs of the eye, can be grouped into the oculomotor system, and a protective structure.

The oculomotor system allows moving the eye towards certain sectors of the environment; its particular organization and its connections with the brain make it suitable to transmit to the analytical neurological centres a complex of signals that faithfully repeat the images of the external world [1].

The eyeball, together with the oculomotor system and the protective apparatus, is located in the orbital cavity that is a deep, bone cavity, formed by the convergence of the processes of the maxillary, the zygomatic, and the palatine bones, in the lower part; by the convergence of the frontal and the sphenoid bones superiorly; from the lacrimal ethmoid and, minimally, maxillary, and sphenoid bones, medially; and from the zygomatic and the sphenoid bones, laterally [1].

The eyeball is contained in a connective structure called Tenon's capsule, which separates it from the orbital fat. It forms a sort of bulb support, for which it has the function of an acetabulum.

The development of visual functions begins in the first months of life and is completed around the one year of age [2].

In this period, the maturation of the retina and the visual pathways occur, and binocular bonds are established at the level of the visual cortex. During this phase of maturation and then-for a few years-a certain plasticity of visual functions remains; therefore, during this "plastic" period, it seems susceptible to change both in a pejorative sense 
(by pathogens) or in an improving sense (for example, by therapeutic measures). This plasticity appears maximum during the first months of life and then gradually decreases until it disappears around the 7th-8th year of life [2].

Moreover, during the "plastic" period of the visual system, the development of its various functions does not occur independently; as a consequence, if a pathological cause alters the development of one of the functions, the other functions may also be affected. In particular, the mechanisms of the motor and the sensory fusions, which occur in the oculomotor system during the first years of life, play an important role.

The term "sensory fusion" indicates the unification of visual excitations from the corresponding retinal images, into a single visual perception (a single image). A subject is unable to see double, if the external object stimulates the retinal points that have the same spatial location, defined as "correspondent" [2, 22].

A "fusionable" stimulus-different from a stimulus that could elicit a retinal rivalry-can appear from the age of 4-5 months of a child $[2,22]$.

The critical period in which the sensory fusion could be compromised lasts until the 6th-7th year of life. During the first two or three years of life, a few weeks of interrupted binocularity is a period able enough to definitively abolish the sensory fusion. After this period, the ocular function gradually tends to become stronger, so that at 5-6 years it could be recovered even after a long period of interruption $[2,22]$.

The term "motor fusion" indicates the ability to align the eyes in order to maintain the "sensory fusion". The stimulus for these fusing eye movements is the retinal disparity. The retinal disparity means the simultaneous stimulation of mismatched retinal elements [2].

In normal conditions, the retinal image disparity produces diplopia. But the fusional movements can trigger a "vergency response" to align the images of the object in regard to the foveas. A positive fusional vergence measures the extent to which a person can maintain the "fusion" and align the eyes, with gradually increasing vergence demands.

In a normal person, the ability to recognize incorrect binocular alignments is limited by the quality of sensory information. In the first 3-4 months of life, the reduced visual acuity, the absence of stereopsis, and the immaturity of cortical neurons greatly limit the movements of fusional convergence [22]. Binocular alignment is therefore very unstable in the first months of life and becomes more accurate around the 5th or 6th month. The mechanism of fusional convergences is variously susceptible to any pathogenic factor up to about 9 years of age of the child [22].

The visual function is strongly interconnected with other functions aimed at the orientation of the individual in the surrounding space. Among these, there are, for example, the positioning of the head with respect to the cervical spine, for the maintenance of an upright posture, and the coordinated movements of the head and eyes for the exploration of the surrounding space. These interconnections are achieved through neurological links, which also involve the stomatognathic apparatus.
Several studies have shown that the stimulation of extraocular muscles induces effects on oculomotor function, and vice versa. For example, the oculomotor function is closely related to information coming from the cervical tract, through the oculocephalic reflex, mediated by afferents from $\mathrm{C} 2$ and $\mathrm{C} 3$ nerves. In the oculocephalic reflex, there is the presence of a triggering ascending stimulus, from the trigeminal nucleus, that carries information to the ocular nuclei and a descending stimulus that carries information to the spinal cord through the medial longitudinal fascicle [23]. Consequently, the simultaneous management of visual and vestibular afferent stimuli in the central nervous system influences the proper position of the head in space, according to the position of the eyes, and to the cervical posture.

Buisseret-Delmas et al. in an experiment, by injecting peroxide into the ocular muscles, observed its diffusion in many areas of the nervous system: the Gasser ganglion, the trigeminal spinal cord, and the cervical spine dorsal horn (C1C2) [24].

A major station for these interconnections is represented by the hypoglossal nerve (XII cephalic nerve) that also gives the motor impulses to the tongue. The hypoglossal nerve is classically considered a pure motor nerve, but it also contains a proprioceptive component. During its extracranial decorsus, it receives the proprioceptive fibres from the cervical plexus (the nerves of the first three cervical vertebrae, $\mathrm{C} 1, \mathrm{C} 2$, and $\mathrm{C} 3$ nerves), thus collecting the proprioception information from the suboccipitals, trapezius, and sternocleidomastoid muscles and from the oculomotor apparatus, besides that of the tongue: consequently, this neurological connection represents a conjunction among the stomatognathic apparatus, the oculomotor system, and the cervical area.

Another major station is represented by the superior colliculus (SC), a structure which coordinates visual, somatic, and auditory information by directing the movements of the head and the eyes towards the source of a stimulus. Among the seven layers that constitute the SC, there are three sensitive maps (a visual map, a somatic map of the body surface, and a map of the spatial location of the sounds), plus a motor map. Consequently, the role of the visual function, in maintaining the balance of the head, or during walking and during all other motor coordination, is related to this structure. About this structure, it was observed in rats that neurons in the trigeminal mesencephalic nucleus (5me) can project to the SC. In addition, the existence of SC projections to neurons in $5 \mathrm{me}$ was reported. In particular, it seems that the SC fibres contact "en passant" small as well as large cell bodies of 5 me neurons. These pathways suggest a role of the 5 me neurons in oculomotor control and associated orofacial functions [25].

The cells of the $5 \mathrm{me}$ are called protoneurons (i.e., neurons of a neuronal chain) constituting the equivalent of a peripheral sensitive ganglion [8] that would explain the "sensitivity" of the stomatognathic apparatus to descending (stress, anxiety, etc.) and ascending stimuli (proprioception of the column, legs, and feet) [26-29].

In addition, the oculi extrinsic muscles, masticatory muscles, dental pulp, and periodontal ligaments seem linked 
by the $5 \mathrm{me}$ to the cerebellum, the reticular formation, the vestibular nuclei, the predominant nucleus, and the spinal motoneurons. The core of these connections constitutes an important centre for calculating the position and the eye movements due to connections with vestibular nuclei, the oculomotor nuclei, and the cerebellum.

On the base of all these concepts, the relationship between the oculomotor and the stomatognathic apparatus is an increasingly interesting subject for researchers in the field of dentistry.

On the base of these observations, a role for trigeminal afferents on body posture was hypothesized, but this has not yet been demonstrated conclusively $[3,4,8]$.

Thus, the aim of this review was to clarify the level of evidence in the scientific literature about the correlations between the visual and the stomatognathic apparatus in human subjects.

\section{Materials and Methods}

In this systematic review, the results of relevant studies are summarized. Data were searched in the PubMed database, Scopus database, and the Cochrane Library using the following keywords: oculomotor, stomatognathic, dental occlusion, correlations, visual defects, vision problems for published or in press studies dated through June 2018. The search strategy is summarized in Table 1.

A hand research was conducted in the reference list of the resulting studies. Based on the titles, abstracts, and full texts of the studies when needed, two researchers dealt with the selection of the studies according to the following criteria: studies on human subjects, no case reports, and studies in English, Italian, Spanish, French, or German. From each of the selected studies, only the results concerning the correlations between the visual function and stomatognathic apparatus are reported. Data on the type and the construction of the study (the design, the presence of a control group, the mechanism of randomization, the method error study, and the technical description of the methods) were also recorded in order to assess their level of evidence. The quality of the studies was established by the assignment of scores to each full-text article included in the qualitative analysis. The quality of each study, with a maximum possible score of 11 , was considered as follows: low: total score $\leq 4$; medium: $5 \leq$ score $\leq 8$; high: score $\geq 9$.

\section{Results}

Out of the total number of 64 articles, 19 were qualified for the final analysis.

The quality level of the studies was judged to be low for 3 studies, medium for 13 studies, not classified for 2 studies, and high for 1 study (Table 2).

Thus, the overall level of the studies on this topic is judged to be merely adequate. The level of quality is mainly affected by the lack, actually in literature, of an adequate number of prospective longitudinal reports that could really clarify the relationship between the visual system and the stomatognathic apparatus.
TABLE 1: Search strategy.

\begin{tabular}{lc}
\hline 1 & Oculomotor \\
\hline 2 & Stomatognathic \\
\hline 4 & Dental occlusion \\
\hline 5 & Visual defects \\
\hline & Vision problems \\
\hline
\end{tabular}

The main results and methods are summarized in Table 3. A part of the studies pointed on the anatomical contiguity between the stomatognathic and visual apparatus. Two of these studies are virtual studies on finite elements analysis $[3,4]$, and there is only one longitudinal prospective study $[5]$.

In summary, these studies emphasize the role of the sphenoid bone for the contiguity between the two areas, because it forms part of the orbit and also is in relation to the maxillary bone $[3,4]$.

The higher scientific evidence is given by the only prospective longitudinal study by Habeeb [5] that showed a statistically significant increase in the ocular interaxial distance between the two eyes of about $0.25 \mathrm{~mm}$ after rapid palatal expansion.

Another part of the studies focuses on the neurological connections between the stomatognathic and the visual apparatus.

These studies are mainly organized as case-control or cross-sectional observational studies [6, 8-21]. Most of the studies are on children $[6,10-21]$. Some studies are on adults, mainly focusing on subjects with temporomandibular disorders $[8,9]$.

\section{Discussion}

The purpose of this study was to review the existing literature data on the correlations between the stomatognathic and the visual apparatus and finally to establish the level of evidence. Studies have been collected from the main databases, based on keywords.

A part of the studies pointed on the anatomical contiguity between the stomatognathic and visual apparatus, and other studies focus on neurological connections.

It is known that the eyeball, together with the oculomotor system and the protective apparatus, is located in the orbital cavity that is a deep, bone cavity, formed by the convergence of the processes of the maxillary, the zygomatic, and the palatine bones, in the lower part; by the convergence of the frontal and the sphenoid bones superiorly; from the lacrimal ethmoid and, minimally, maxillary, and sphenoid bones, medially; and from the zygomatic and the sphenoid bones, laterally [1].

The eyeball is thus contained in a connective structure called Tenon's capsule, which separates it from the orbital fat. It forms a sort of bulb support, for which it has the function of an acetabulum. 


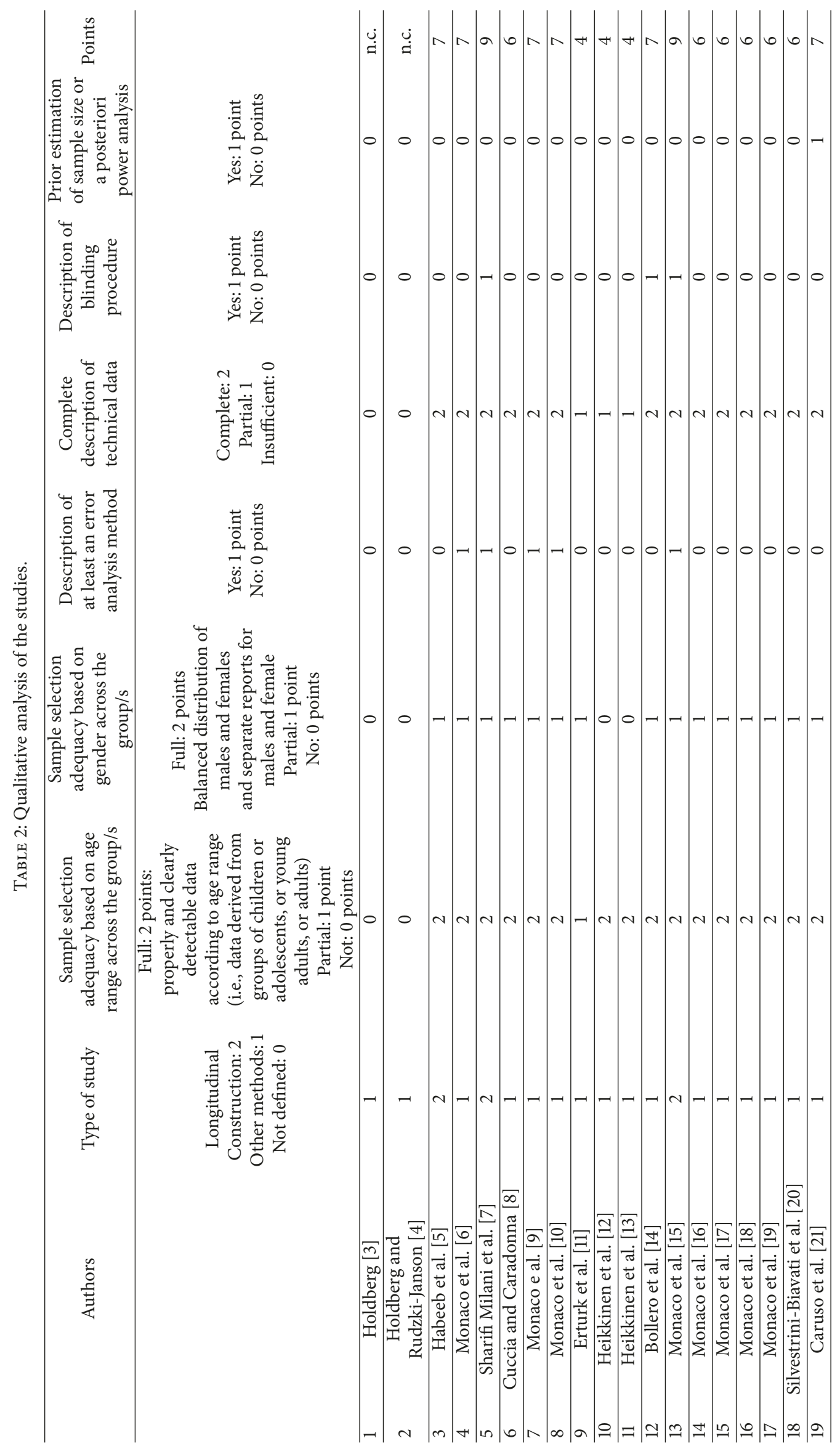




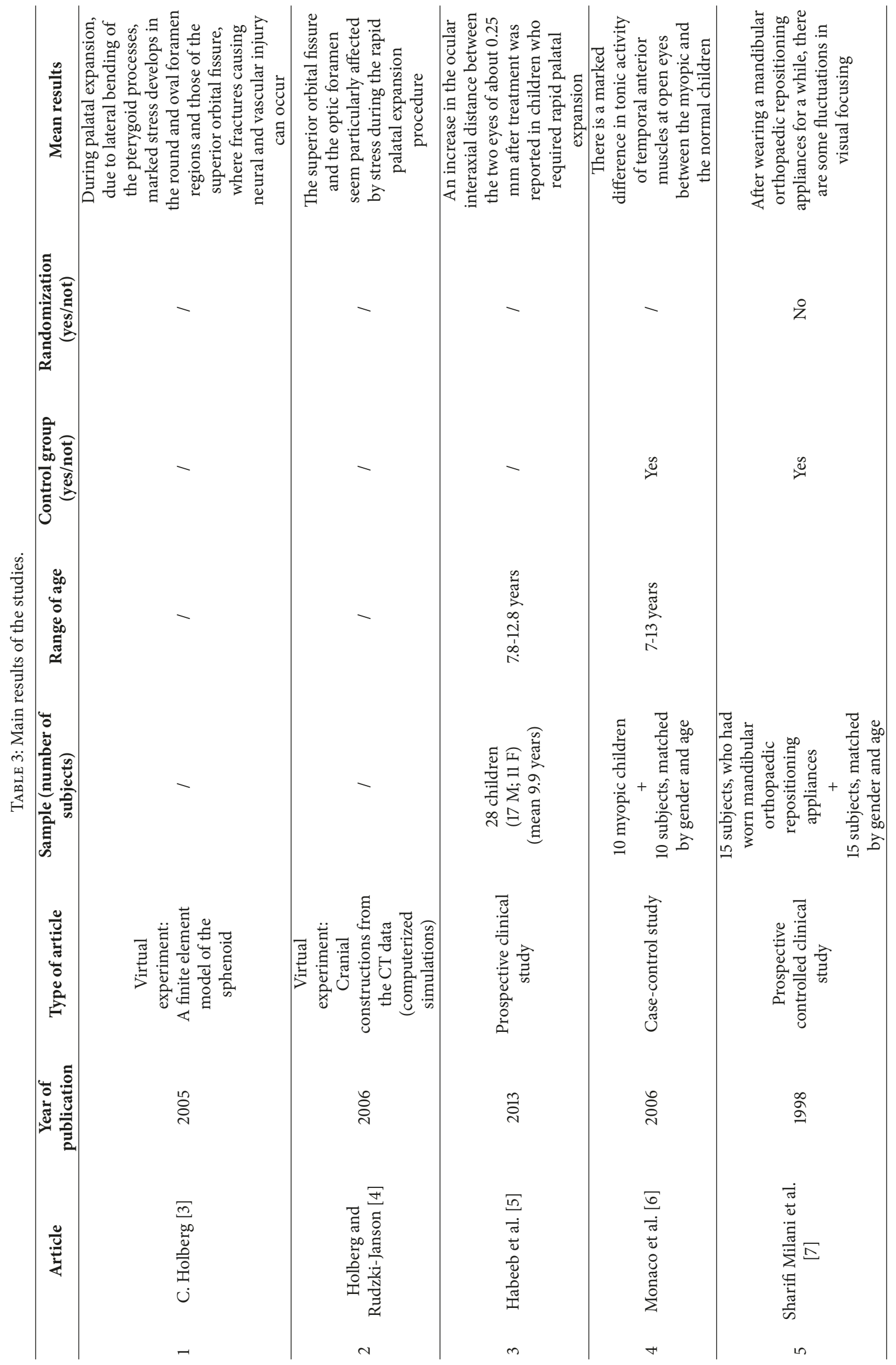




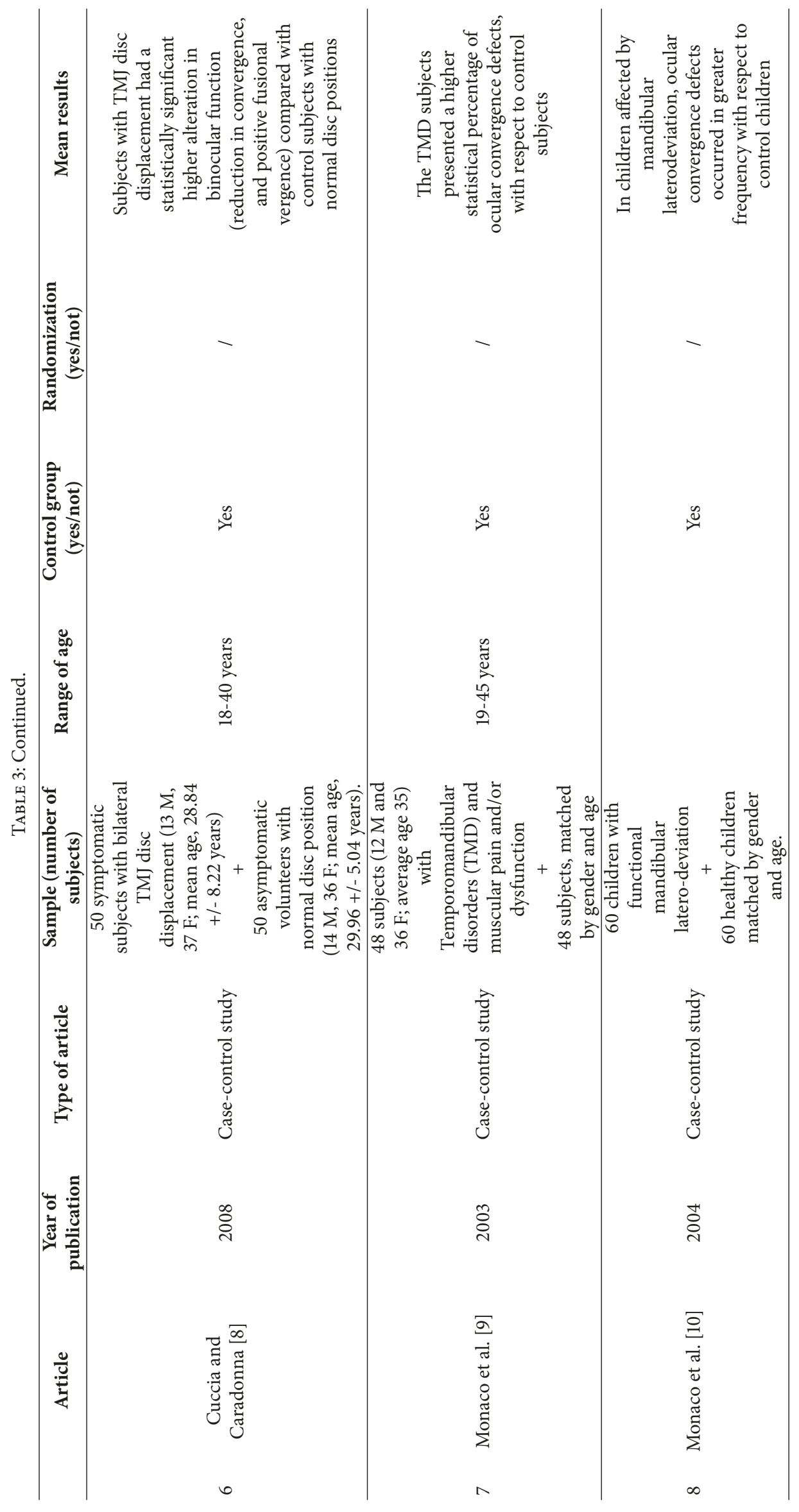




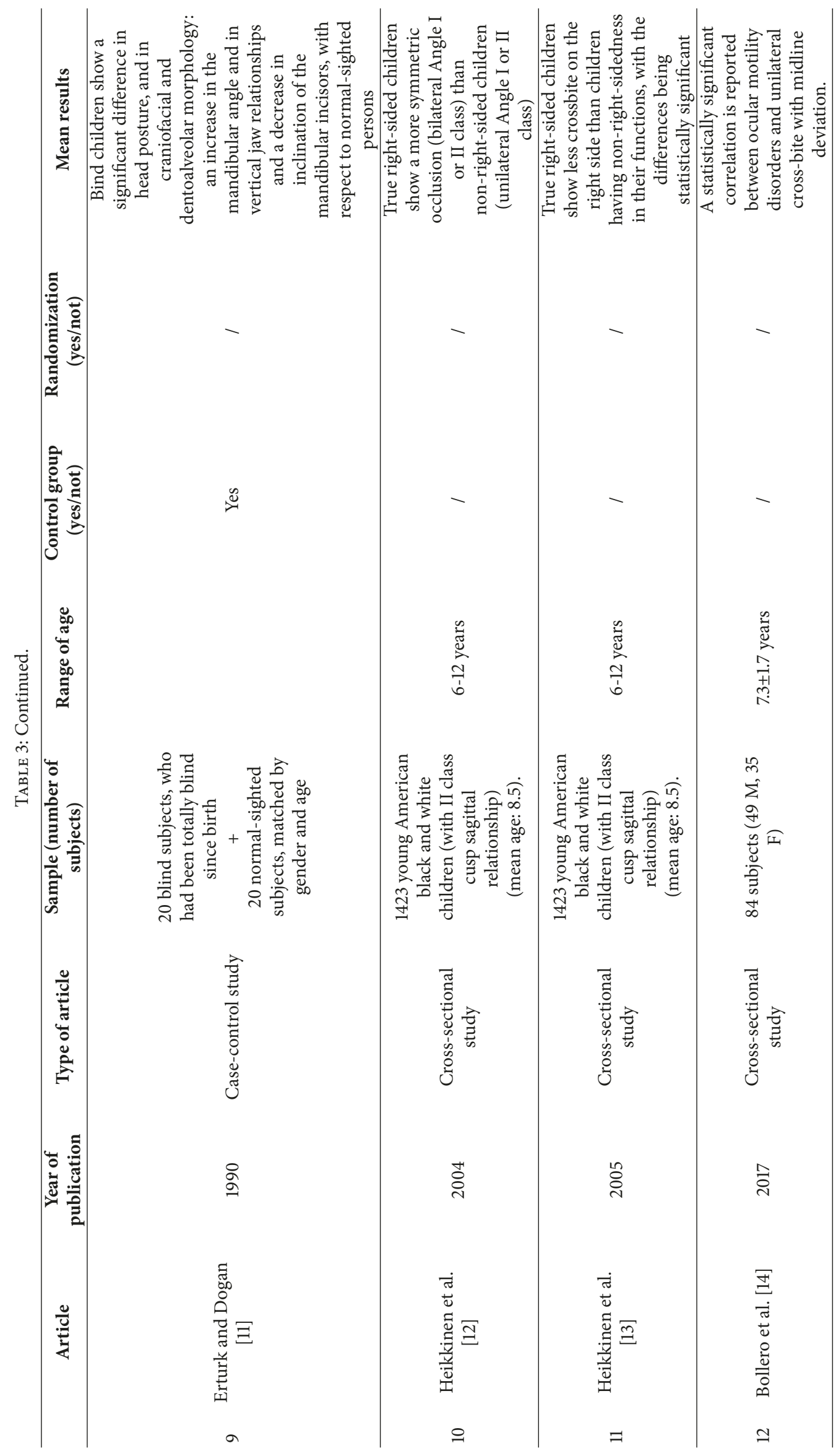




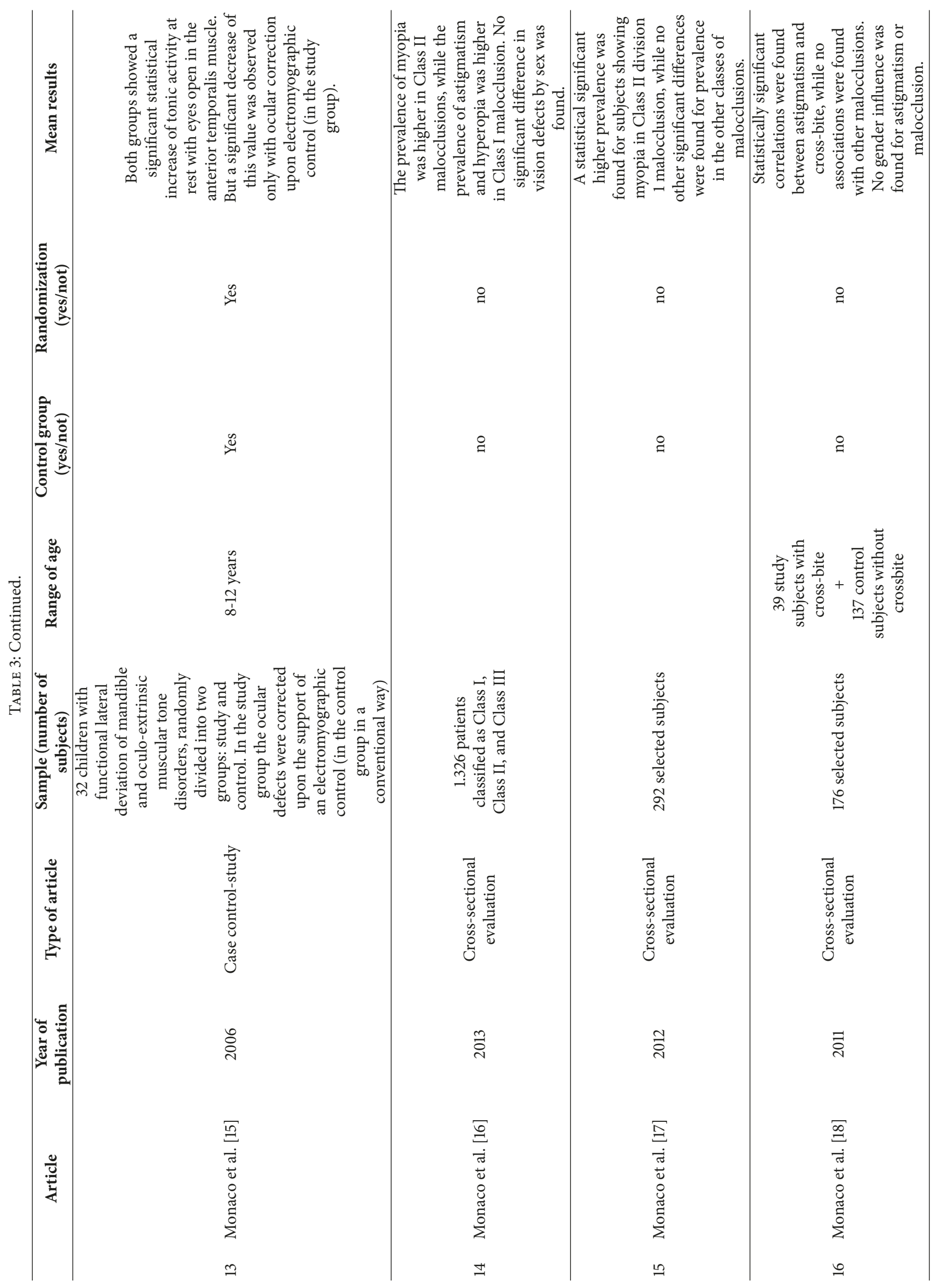




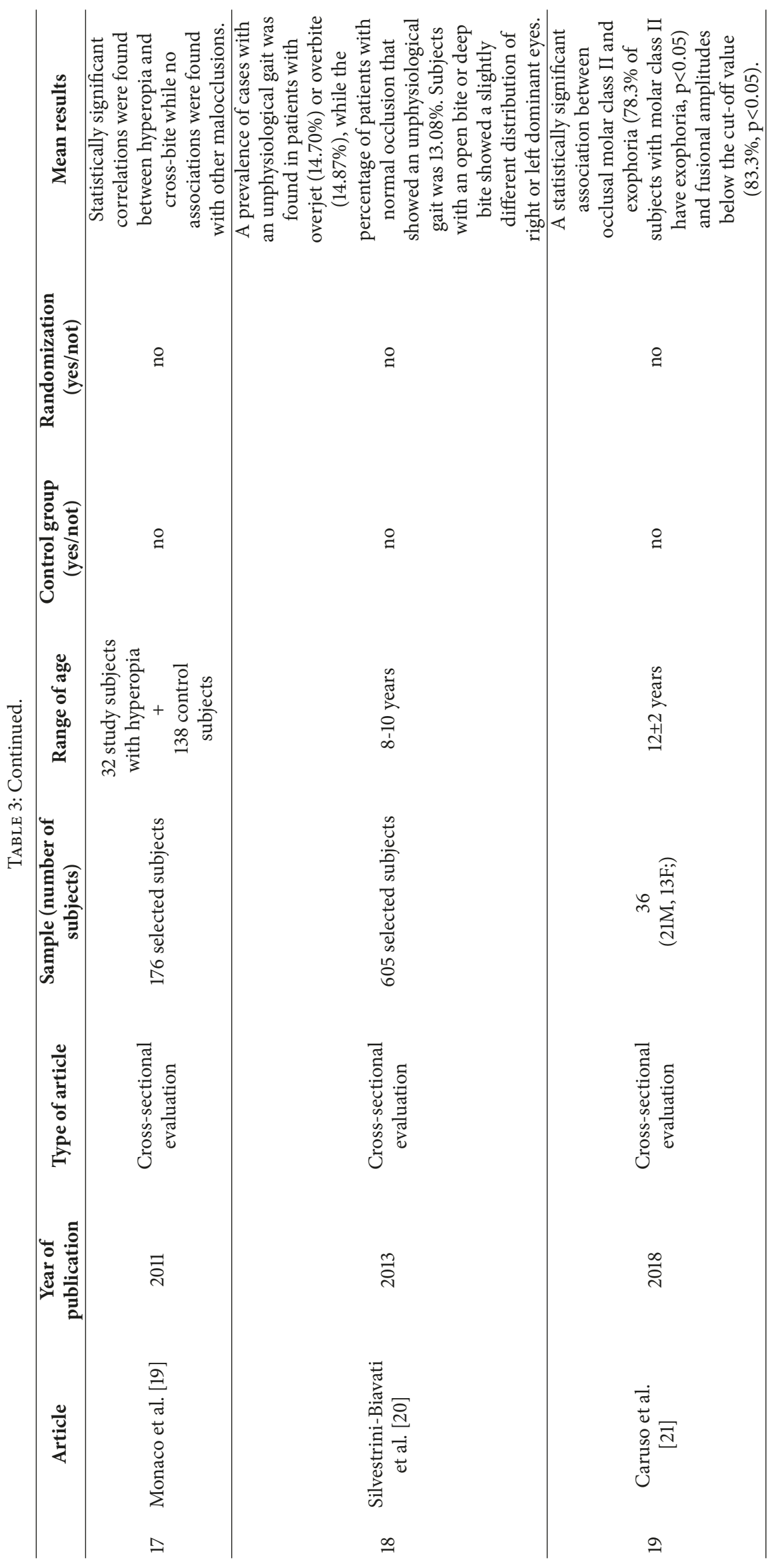


About the anatomic contiguity, this review clarifies that there is an effect on the visual apparatus after a procedure of palatal expansion. It seems that the palatal expansion is accompanied by an increase of the stress at the sphenoid bone, which could determine an increase of the interorbital distance of $0.25 \mathrm{~mm}$ in children, but the clinical importance of this finding is yet to be clarified.

The evidence is given by three studies; two of them are virtual studies.

In one of them, Holberg and Rudzki-Janson [4] evaluate the stress distribution induced by a palatal expander on the sutures of the cranial base through a finite elements analysis, simulating data for children and adults, and report that the greatest stress is located in the sphenoid bone that, through the pterygoid processes, is in continuity with the jawbone. In particular, the higher stress due to the palatal expander was located in the pterygoid processes and the boreholes: the superior orbital fissure and the optic foramen seem particularly affected. In addition, it was proved by Holberg [3] that this stress could lead to alterations of the oculomotor function, through a direct mechanism-changing the position of the optical hole that attaches to the Zinn tendon ring that is the common origin of 5 oculomotor muscles-and/or through an indirect mechanism, compressing one or more orbital nerves.

Although a virtual analysis can provide only a theoretical scientific evidence of relationship, the conclusions of these studies are also supported in literature by a case-report of an adult by the experience of Lanigan and Mintz [30] that describe the paresis of the ocular nerve after a surgically assisted palatal expansion, observing by tomography that the cause of this complication was a small fracture of the sphenoid.

About the anatomical contiguity, the higher scientific evidence is given by the only prospective longitudinal study by Habeeb et al. [5] that showed a statistically significant increase in the ocular interaxial distance between the two eyes of about $0.25 \mathrm{~mm}$ after rapid palatal expansion (in a sample of 28 children, 17 boys and 11 girls, mean age, 9.9 years), but the clinical significance of this observation remains indefinite. A clinical consequence is that the clinician can realize that with activation of the rapid maxillary expansion appliance he/she is producing not only an expansion force at the intermaxillary suture but also forces on other structures within the craniofacial complex that may or may not be beneficial for the patient [31].

About the neurological connections, most of the studies correlate the different visual defects to specific malocclusions.

The ocular convergence defects were associated with functional mandibular lateral deviation in children [10].

The ocular motility disorders were associated with unilateral crossbite with midline deviation in children in a sample of children [14] while fusional vergence defects (exophoria) were associated with molar class II malocclusions [21].

The myopia in children was associated with the presence of crossbite [18] and with the presence of class II, division I malocclusions $[16,17]$. In addition, about the myopia, a pair of case-control studies report an evident association between myopia and the tonic activity of the anterior temporalis muscle. In a case-control comparison, myopic children (a sample of 10 subjects, 7-13 years old) showed a marked difference in the tonic activity of anterior temporalis muscle at open eyes, with respect to children with normal vision (10 matched subjects) [6].

In addition, a correction of the oculomotor defect programmed upon an electromyographic control of the anterior temporalis muscle resulted accompanied by a statistically significant decrease of the tonic activity of this muscle at open eyes-almost in children with oculomotor defects and mandibular functional lateral deviation-with respect to children that received a conventional visual correction (casecontrol study) [15].

The hyperopia in children was associated with the presence of a crossbite [19] and with the presence of class I malocclusions $[16,17]$.

The astigmatism was associated with the presence of class I malocclusions $[16,17]$.

The ocular right-sided condition in children was associated with a more symmetric dental occlusion (bilateral class I or II condition) [12], with respect to the not rightsidedness condition, in a cross-sectional evaluation. The ocular right-sided condition in children was also related to a lower prevalence of crossbites on the right side, with respect to the condition with non-right-sidedness in their function [13].

The presence of an unphysiological gait due to ocular convergence defects was associated with increased overjet and overbite [20].

A pair of studies focused on subjects with temporomandibular joint disorders (TMD) in the adult population, and some associations were also observed between TMD and oculomotor function in adults. Furthermore, a considerably higher prevalence of ocular convergence defects was assessed in adults affected by TMD presenting limited maximal opening, myofascial pain, and pain in the neck shoulder area, with respect to healthy individuals [9].

In addition, in a case-control study on 50 patients affected by TMD, compared with an equal number of control subjects, it was observed a significantly lower binocular function, measured as convergence and positive fusional vergence, with respect to control healthy subjects with normal disc position [8].

But the higher scientific evidence is related to a prospective controlled clinical study conducted by Sharifi Milani and Coll. [7], which reports a correlation between dental occlusion and visual focus in children treated with a jaw orthopaedic repositioning device. This device, especially in children with occlusal disarming, caused changes in the focus ability that disappeared by removing the device itself. In this study thirty subjects were divided into two groups: an experimental group treated with mandibular orthopaedic repositioning appliances and an untreated control group. All of the subjects underwent the same visual focusing tests with a Maddox rod and the Berens prismatic bars, from over five meters to 30 centimetres. The results showed that the alteration of dental occlusion can induce some fluctuations in visual focusing. The phenomenon occurred after wearing a mandibular orthopaedic repositioning appliance for a while. 
Feedback effects were gradual after removing the mandibular splint.

As the great part of the studies are based on a case-control or a cross-sectional construction, only some hypotheses were possible to explain the reasons for these types of correlations.

The great part of the researchers seem in agreement to assume that the nociceptive stimuli due to the inflammation of masticatory muscles or temporomandibular joint following malocclusion in children may cause some alterations in nerve conduction (such as hyperexcitability, temporal aggregation, and glial cell activation) so as to determine a "central fatigue" and therefore induce recruitment of fewer muscle fibres for eye movements: this could explain the alteration of binocular mobility [32].

These assumptions seem to be supported by a casecontrol study published by Erturk and Dogan [11] that compared craniofacial and dentoalveolar morphology in blind children and control healthy children. Their results revealed a change in the inclination of the head and a reduction in the size of the upper jaw in blind children, the latter probably related to the insufficient development of the orbital region that was detected. The same authors also hypothesized a correlation between the crown dimension of deciduous teeth and strabismus and suggested that strabismus can result from child developmental changes and that the resulting asymmetries may involve the whole head, including the teeth.

\section{Conclusions}

The purpose of this study was to review the existing literature data on the correlations between the stomatognathic and the visual apparatus and finally to establish the level of evidence.

As the great part of the studies in this field are observational case-control or cross-sectional analyses, their level of evidence allows establishing that there is a correlation between ocular disorders (myopia, hyperopia, astigmatism, exophoria, and an unphysiological gait due to ocular convergence defects) and dental occlusion, but it is not possible to establish the cause-effect relationship.

Furthermore, in adult samples, a considerably higher prevalence of ocular convergence defects was assessed in adults affected by TMD.

A slight evidence, based on very few data, allows us to establish that intraoral appliances could change ocular ability: it seems that there is an increase of ocular interaxial distance between the two eyes of about $0.25 \mathrm{~mm}$ after a rapid palatal expansion in children; and in addition a mandibular anterior repositioning appliance, especially in children with occlusal disarming, seems to cause changes in the focus ability that disappear by removing the device itself.

But these evidences are based only on small samples.

Consequently, future studies should be aimed at establishing higher levels of evidence (prospective, controlled, and randomized studies).

\section{Conflicts of Interest}

The authors declare that there are no conflicts of interest regarding the publication of this paper.

\section{References}

[1] J. A. Juanes, P. Ruisoto, A. Prats-Galino, A. Framiñán, and J. M. Riesco, "Computed anatomical modelling of the optic pathway and oculomotor system using magnetic resonance imaging," Journal of Neuroradiology, vol. 41, no. 3, pp. 168-176, 2014.

[2] C. E. Peeler, "A Review of Visual and Oculomotor Outcomes in Children With Posterior Fossa Tumors," Seminars in Pediatric Neurology, vol. 24, no. 2, pp. 100-103, 2017.

[3] P. Gangloff, J.-P. Louis, and P. P. Perrin, "Dental occlusion modifies gaze and posture stabilization in human subjects," Neuroscience Letters, vol. 293, no. 3, pp. 203-206, 2000.

[4] A. Baldini, A. Nota, G. Cravino, C. Cioffi, A. Rinaldi, and P. Cozza, "Influence of vision and dental occlusion on body posture in pilots," Aviation, Space, and Environmental Medicine, vol. 84, no. 8, pp. 823-827, 2013.

[5] C. Holberg, "Effects of rapid maxillary expansion on the cranial base - An FEM-analysis," Journal of Orofacial Orthopedics, vol. 66, no. 1, pp. 54-66, 2005.

[6] A. Monaco, O. Streni, M. C. Marci, L. Sabetti, and M. Giannoni, "Convergence defects in patients with temporomandibular disorders," The Journal of Craniomandibular \& Sleep Practice, vol. 21, no. 3, pp. 190-195, 2003.

[7] D. T. Lanigan and S. M. Mintz, "Complications of surgically assisted rapid palatal expansion: Review of the literature and report of a case," Journal of Oral and Maxillofacial Surgery, vol. 60, no. 1, pp. 104-110, 2002.

[8] A. M. Cuccia and C. Caradonna, "Binocular motility system and temporomandibular joint internal derangement: A study in adults," American Journal of Orthodontics and Dentofacial Orthopedics, vol. 133, no. 5, pp. 640-e20, 2008.

[9] M. Habeeb, N. Boucher, and C.-H. Chung, "Effects of rapid palatal expansion on the sagittal and vertical dimensions of the maxilla: A study on cephalograms derived from cone-beam computed tomography," American Journal of Orthodontics and Dentofacial Orthopedics, vol. 144, no. 3, pp. 398-403, 2013.

[10] N. Ertürk and S. Doğan, "The effect of neuromuscular diseases on the development of dental and occlusal characteristics," Quintessence International, vol. 22, no. 4, pp. 317-321, 1991.

[11] C. Holberg and I. Rudzki-Janson, "Stresses at the cranial base induced by rapid maxillary expansion," The Angle Orthodontist, vol. 76, no. 4, pp. 543-550, 2006.

[12] A. Monaco, F. Sgolastra, R. Cattaneo et al., "Prevalence of myopia in a population with malocclusions," European Journal of Paediatric Dentistry, vol. 13, no. 3, pp. 256-258, 2012.

[13] A. Monaco, F. Sgolastra, A. Petrucci, I. Ciarrocchi, P. D. D'Andrea, and S. Necozione, "Prevalence of vision problems in a hospital-based pediatric population with malocclusion," Journal of Pediatric Dentistry, vol. 35, no. 3, pp. 272-274, 2013.

[14] T. Heikkinen, T. Poikela, M. Grön, and L. Alvesalo, "Unilateral Angle II in functional lateralities," European Journal of Orthodontics, vol. 26, no. 1, pp. 93-98, 2004.

[15] A. Monaco, O. Streni, M. C. Marci, L. Sabetti, G. Marzo, and M. Giannoni, "Relationship between mandibular deviation and ocular convergence," Journal of Clinical Pediatric Dentistry, vol. 28, no. 2, pp. 135-138, 2004.

[16] A. Monaco, A. Spadaro, F. Sgolastra, A. Petrucci, P. D. D’Andrea, and R. Gatto, "Prevalence of hyperopia and strabismus in a paediatric population with malocclusions," European Journal of Paediatric Dentistry, vol. 12, no. 4, pp. 272-274, 2011.

[17] A. Monaco, A. Spadaro, F. Sgolastra, A. Petrucci, P. D. D’Andrea, and R. Gatto, "Prevalence of astigmatism in a paediatric 
population with malocclusions," European Journal of Paediatric Dentistry, vol. 12, no. 2, pp. 91-94, 2011.

[18] A. Monaco, R. Cattaneo, A. Spadaro, M. Giannoni, S. Di Martino, and R. Gatto, "Visual input effect on EMG activity of masticatory and postural muscles in healthy and in myopic children.," European journal of paediatric dentistry., vol. 7, no. 1, pp. 18-22, 2006.

[19] A. Monaco, R. Cattaneo, A. Spadaro, P. D’Andrea, G. Marzo, and R. Gatto, "Ocular correction effects on EMG activity of stomatognathic muscles in children with functional mandibular lateral- deviation: a case control study.", European Journal of Paediatric Dentistry, vol. 7, no. 2, pp. 81-88, 2006.

[20] T. Heikkinen, P. Pirttiniemi, M. Grön, and L. Alvesalo, "Primary dentition unilateral crossbite in relation to functional lateralities," Journal of Dentistry for Children, vol. 72, no. 2, pp. 81-87, 2005.

[21] P. Bollero, M. R. Ricchiuti, G. Laganà, G. Di Fusco, R. Lione, and P. Cozza, "Correlations between dental malocclusions, ocular motility, and convergence disorders: A cross-sectional study in growing subjects," ORAL and Implantology, vol. 10, no. 3, pp. 289-294, 2017.

[22] J. A. Büttner-Ennever, "Mapping the oculomotor system," Progress in Brain Research, vol. 171, pp. 3-11, 2008.

[23] P. Buisseret, "Primary projection of receptors of extrinsic ocular muscles: anatomical results," Agressologie, vol. 32, no. 3, pp. 178179, 1991.

[24] C. Buisseret-Delmas, C. Compoint, C. Delfini, and P. Buisseret, "Organisation of reciprocal connections between trigeminal and vestibular nuclei in the rat," Journal of Comparative Neurology, vol. 409, no. 1, pp. 153-168, 1999.

[25] A. Ndiaye, G. Pinganaud, F. VanderWerf, C. Buisseret-Delmas, and P. Buisseret, "Connections between the trigeminal mesencephalic nucleus and the superior colliculus in the rat," Neuroscience Letters, vol. 294, no. 1, pp. 17-20, 2000.

[26] A. Nota, S. Tecco, S. Ehsani, J. Padulo, and A. Baldini, "Postural stability in subjects with temporomandibular disorders and healthy controls: A comparative assessment," Journal of Electromyography \& Kinesiology, vol. 37, pp. 21-24, 2017.

[27] A. Baldini, A. Nota, C. Cioffi, F. Ballanti, and S. Tecco, "Mandibular position influence on pilots' postural balance analyzed under dynamic conditions," Cranio: Journal of Craniomandibular Practice, vol. 35, no. 6, pp. 367-371, 2017.

[28] S. Tecco, G. Farronato, V. Salini et al., "Evaluation of cervical spine posture after functional therapy with FR-2: A longitudinal study," The Journal of Craniomandibular \& Sleep Practice, vol. 23, no. 1, pp. 53-66, 2005.

[29] S. Tecco, S. Caputi, and F. Festa, "Evaluation of cervical posture following palatal expansion: A 12-month follow-up controlled study," European Journal of Orthodontics, vol. 29, no. 1, pp. 4551, 2007.

[30] A. Silvestrini-Biavati, M. Migliorati, E. Demarziani et al., "Clinical association between teeth malocclusions, Wrong posture and ocular convergence disorders: An epidemiological investigation on primary school children," BMC Pediatrics, vol. 13, no. 1, article no. 12, 2013.

[31] S. Caruso, R. Gatto, M. Capogreco, and A. Nota, "Association of Visual Defects and Occlusal Molar Class in Children," BioMed Research International, vol. 2018, Article ID 7296289, 4 pages, 2018.

[32] A. Jafari, K. S. Shetty, and M. Kumar, "Study of stress distribution and displacement of various craniofacial structures following application of transverse orthopedic forces - A threedimensional FEM study," The Angle Orthodontist, vol. 73, no. 1, pp. 12-20, 2003. 


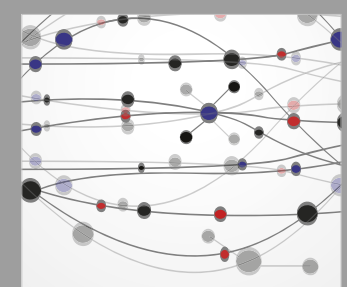

The Scientific World Journal
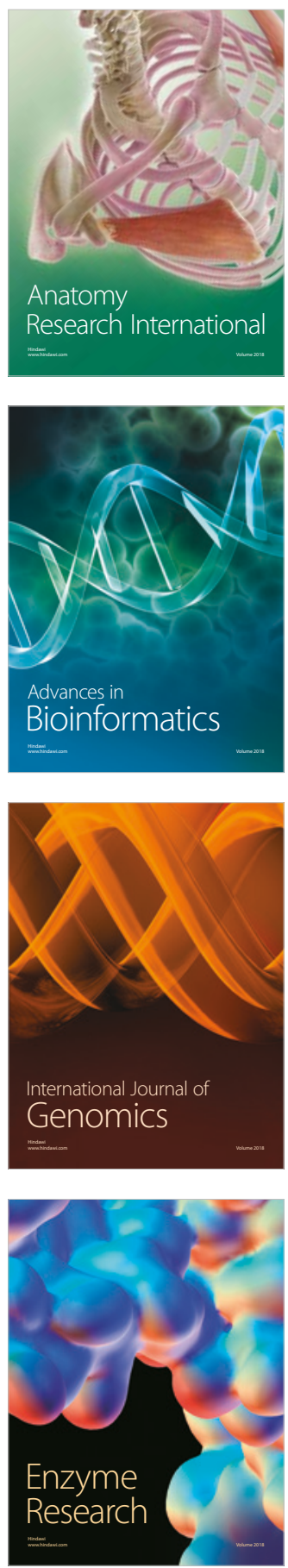
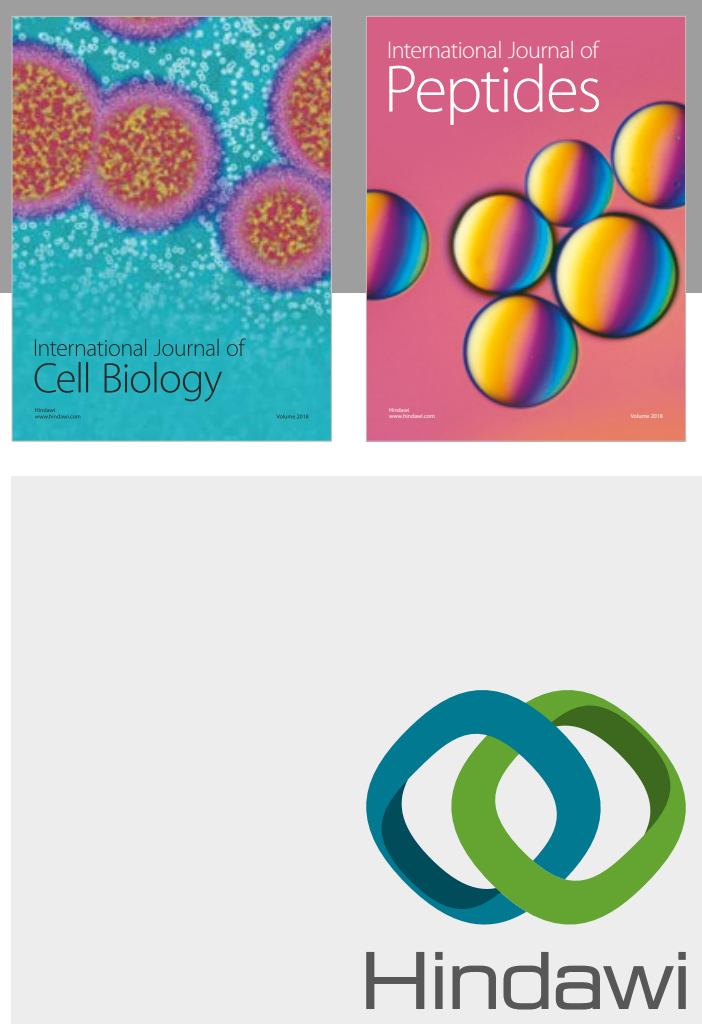

Submit your manuscripts at

www.hindawi.com
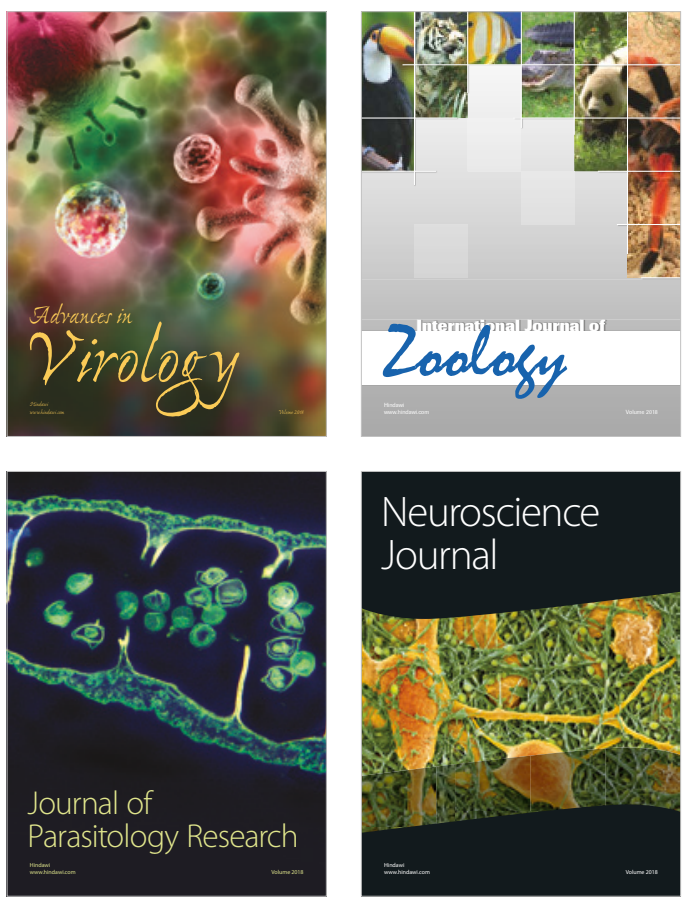
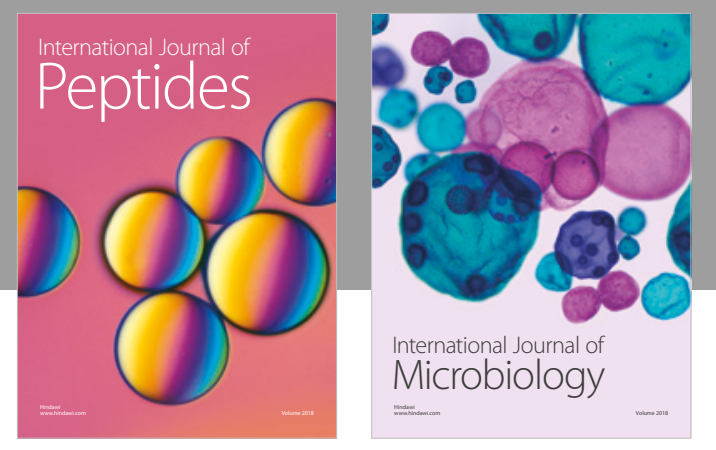

nternational Journal of Microbiology
Journal of
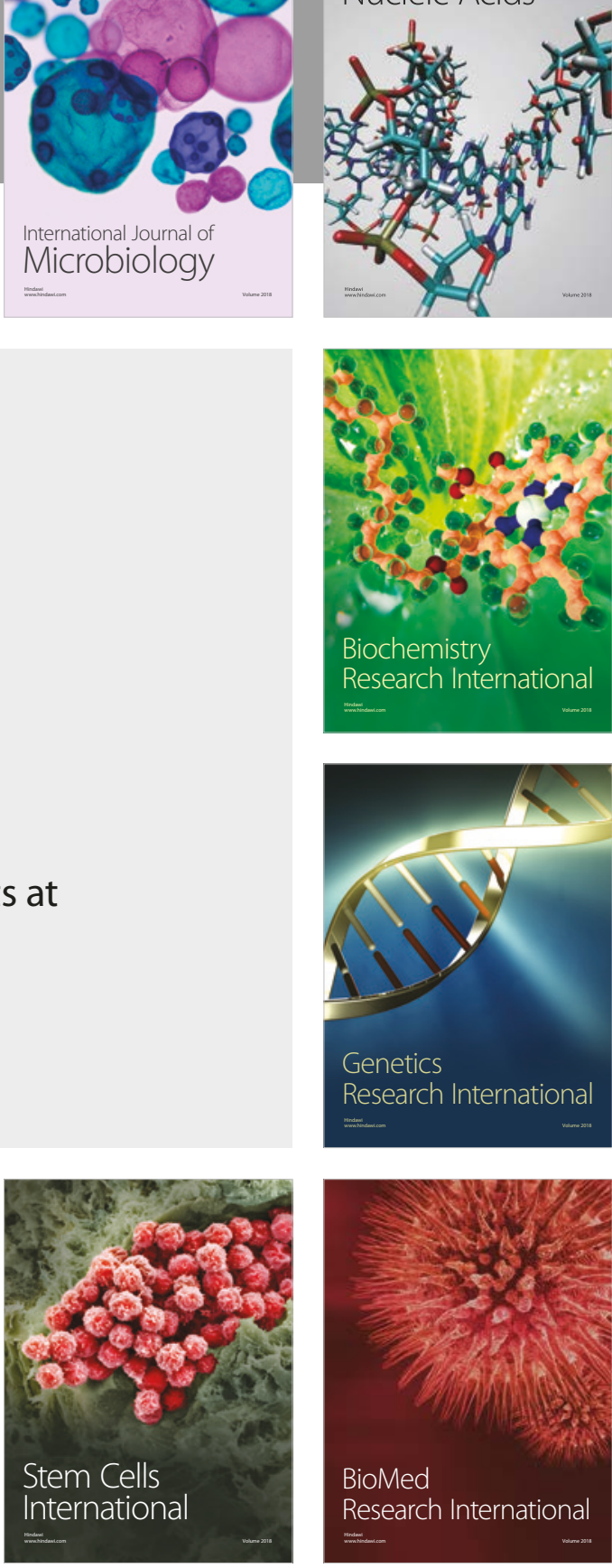
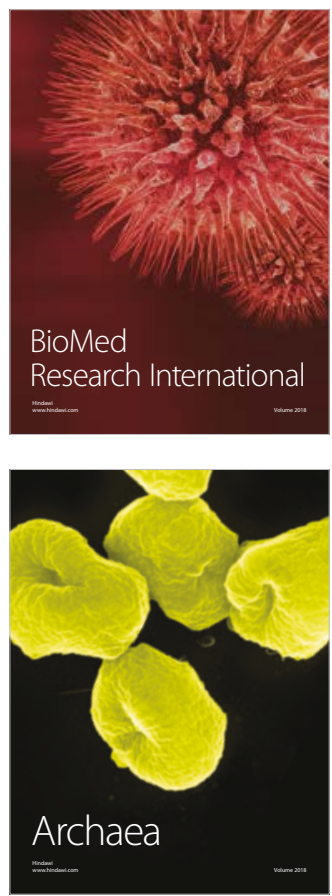